\section{Biological synthesis of cobalt ferrite nanoparticles}

\author{
Anal K. Jha, ${ }^{1}$ Kamal Prasad ${ }^{2}$ \\ 'University Department of Chemistry, \\ T.M. Bhagalpur University, Bhagalpur; \\ ${ }^{2}$ Centre for Applied Physics, Central \\ University of Jharkhand, Brambe, Ranchi, \\ India
}

\begin{abstract}
A low-cost green and reproducible yeast (Saccharomyces cerevisiae) mediated biosynthesis of cobalt ferrite nanoparticles is reported. The synthesis is performed at close to room temperature in the laboratory. X-ray, Fourier transform infrared spectroscopy and high resolution transmission electron microscopy analyses are performed to ascertain the formation of cobalt ferrite nanoparticles. Individual nanoparticles, as well as a very few aggregate having the size of $3-15 \mathrm{~nm}$, were found. The vibrating sample magnetometer measurement showed superparamagnetic behavior in cobalt ferrite nanoparticles. The mechanism involved in the biosynthesis of cobalt ferrite nanoparticles has also been discussed.
\end{abstract}

\section{Introduction}

Nanoscience and technology has been observing an exponential upsurge in all spheres of life. Synthesis of novel materials with a broader spectrum of application has been gaining momentum due to their unique functional properties and their wide variety of potential applications in high-density magnetic recording, ${ }^{1-4}$ ferrofluid technology, ${ }^{5}$ biomedical drug delivery ${ }^{6,7}$ and magnetic resonance imaging, ${ }^{8,9}$ data storage, biosensors, ${ }^{10}$ biocompatible magnetic nanoparticles for cancer treatment, ${ }^{11-14}$ and magneto-optical devices, ${ }^{15-17}$ among others. Cobalt ferrite nanoparticles have been extensively studied because of their high coercitivity, high magnetocrystalline anisotropy and their moderate saturation magnetization. ${ }^{18}$ The cobalt ferrite compound belongs to the spinel structure family with the general formula $\mathrm{AB}_{2} \mathrm{O}_{4}$ which crystallizes in the well-known spinel structure which presents two possible arrangements: the normal spinel and the inverse spinel structures.

Furthermore, in modern nanoscience and technology, the interaction between inorganic molecules and biological structures is one of the most exciting areas of research. It is now well established that many organisms can produce inorganic materials either on an intra- or extra-cellular level. In order to meet the requirements and exponentially growing technological demand, there is a need to develop an eco-friendly approach for nanomaterial synthesis that does not use toxic chemicals in the synthesis protocol. In the present work, baker's yeast (Saccharomyces cerevisiae) was used to assess its potential as putative candidate bacterial and fungal genera for the synthesis of cobalt ferrite nanoparticles (n-CoF). Furthermore, we have tried to explore and establish a cost effective, environmentally friendly and easily reproducible approach for the purpose of scaling up and subsequent downstream processing. The $\mathrm{n}-\mathrm{CoF}$ obtained was characterized by structure [X-ray diffraction (XRD)], morphology [high-resolution transmission electron microscopy (HRTEM)] and magnetic [vibrating sample magnetometer (VSM)] studies. An effort has also been made to understand the mechanism of nanotransformation of accomplishing biosynthesis at the extra-cellular level.

\section{Experimental}

\section{Synthesis of $\mathrm{CoFe}_{2} \mathrm{O}_{4}$ nanoparticles using yeast}

Yeast cells were allowed to grow as suspension culture in $250 \mathrm{~mL}$ sterile distilled water in the presence of suitable carbon ( $2 \mathrm{~g}$ glucose) and nitrogen ( $0.2 \mathrm{~g}$ glycine) source for $36 \mathrm{~h}$. This was treated as source culture. A small portion of this $(25 \mathrm{~mL})$ was filtered and diluted four times by adding 30\% Et-OH containing nutrients ( $1 \mathrm{~g}$ glucose and $0.1 \mathrm{~g}$ glycine). This diluted culture was again allowed to incubate for another $24 \mathrm{~h}$ until it turned a light straw colour (Figure 1). Before being charged the incubation mixture was heated up to $60^{\circ} \mathrm{C}$ till its nutrients were completely dissolved and it turned golden yellow color and a pH value of 6 . Now, $20 \mathrm{~mL}$ of $0.25 \mathrm{M}$ Cobalt nitrate solution was taken and mixed with an equal volume of ferrous sulfate solution with equal molarity and heated for 5-10 min until it turned a light brown color. This solution was charged to the culture solution $(\mathrm{pH} 6)$ and it was heated in a steam bath up to $70^{\circ} \mathrm{C}$ for $10-20$ min until a fluffy chocolate brown deposition started to appear at the bottom of the flask, indicating the initiation of transformation. The culture solution was then cooled and allowed to incubate at room temperature in the laboratory overnight. Next day, the culture solution was observed to have distinct markedly coalescent deep brown clusters deposited at the bottom of the flask leaving the colloidal supernatant at the top (Figure 1).
Correspondence: Kamal Prasad, Centre for Applied Physics, Central University of Jharkhand, C.T.I. Campus, Brambe, Ranchi - 835205, India. Mob. +91.9431.690.360.

E-mail: k.prasad65@gmail.com;

k_prasad65@yahoo.co.in

Key words: nanoparticles, nanomaterials, cobalt ferrite, green synthesis, biosynthesis.

Contributions: AKJ, conception and design; drafting and revising the article for important intellectual content; KP, analysis and interpretation of data, revising article critically for important intellectual content, final approval of the version to be published.

Conflict of interests: the authors declare no potential conflict of interests.

Acknowledgements: authors gratefully acknowledge Dr. G.S. Saini, Advanced Research Instrumentation Centre, Jawaharlal Nehru University, New Delhi, India for providing the TEM images and Dr. K. Amar Nath, HEG Ltd., Bhopal, India for arranging the XRD data.

Received for publication: 13 January 2012.

Revision received: 5 July 2012.

Accepted for publication: 6 July 2012 .

This work is licensed under a Creative Commons Attribution NonCommercial 3.0 License (CC BYNC 3.0).

(c)Copyright A.K. Jha and K. Prasad, 2012

Licensee PAGEPress, Italy

Nanotechnology Development 2012; 2:e9

doi:10.4081/nd.2012.e9

\section{Characterization}

The formation of n-CoF was checked by XRD technique using a X-ray diffractometer (Phillips PW1710, Amsterdam, The Netherlands) with $\mathrm{CuK}_{\alpha}$ radiation $\lambda=1.5406 \AA$ over a wide range of Bragg angles $\left(15^{\circ} \leq 2 \theta \leq 65^{\circ}\right)$. The $2 \theta$ vs. intensity data obtained from this experiment were plotted using the WinPLOTR program and the angular positions of the peaks were obtained with the same program. The unit cell, hkl values and space group were measured using the TREOR program in the FullProf 2000 software and then refined using FullProf profile matching. The Bragg peaks were modeled with pseudo-Voigt function and the background was estimated by linear interpolation between selected background points. The crystallite size $(D)$ and the lattice strain of back propagation neural network were estimated by analyzing the broadening of X-ray diffraction peaks, using the Williamson-Hall approach.

$B \cos \theta=(K \lambda D)+2(\Delta \xi / \xi) \sin \theta$ 
where $B$ is diffraction peak width at half intensity (FWHM) and $\Delta \xi / \xi$ is the lattice strain and $K$ is the Scherrer constant (0.89). The term $K \lambda D$ represents the Scherrer particle size distribution. HRTEM image and lattice fringes of $\mathrm{n}$ - $\mathrm{CoF}$ was obtained using Bruker high resolution transmission electron microscope operated at an accelerated voltage of $200 \mathrm{keV}$. The specimen was suspended in distilled water, dispersed ultrasonically to separate individual particles, and one or two drops of the suspension were deposited onto holey-carbon coated copper grids and dried under infrared lamp. The Fourier transform infrared (FTIR) spectrum was recorded using a high resolution FTIR 2000 Perkin-Elmer infrared spectrophotometer in mid-IR range of $400-4400 \mathrm{~cm}^{-1}$. The magnetization behavior of the prepared sample was studied by a VSM (model PAR-155).

\section{Results and Discussion}

\section{Crystal structure analysis}

Rietveld refinements on the XRD data were carried out on $\mathrm{n}-\mathrm{CoF}$, selecting the space group $P m 3 m$. The XRD (observed, calculated and difference XRD profiles) pattern of n-CoF synthesized using Saccharomyces cerevisiae is shown in Figure 2. It can be seen that the observed and calculated profiles match perfectly $\left(\chi^{2}=0.6827\right)$. The profile fitting procedure adopted was that minimizing the $\chi^{2}$ function. ${ }^{19}$ The XRD analyses indicated that $\mathrm{n}-\mathrm{CoF}$ has a cubic unit cell. The crystal data and refinement factors of $n-C o F$ obtained from XRD data are shown in Table 1. The XRD data agree quite well with the standard values for $\mathrm{CoFe}_{2} \mathrm{O}_{4}$ (ICDD: \#22-1086). A small difference $(0.0465 \AA)$ between the cell parameter of bulk and nanoparticles has been observed. Consequently, a lowering in unit cell volumes from $590.99 \AA^{3}$ (for bulk) to $581.035 \AA^{3}$ (for $\mathrm{n}-\mathrm{CoF}$ ) has been noticed. This may be due to the nanosizing effect in $n-C o F$.

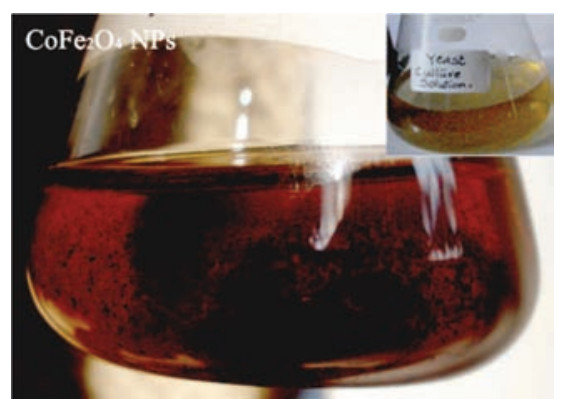

Figure 1. Coalescent dark brown clusters of cobalt ferrite nanoparticles and colloidal supernatant at the top. Inset: yeast culture source.
Figure 3 illustrates the Williamson-Hall plot for $\mathrm{n}$-CoF. A linear least square fitting to $B \cos \theta-\sin \theta$ data yielded $21 \mathrm{~nm}$ average crystallite size and 0.0017 lattice strain, respectively. The residual plot clearly indicates that the error of fitting is well within $\pm 5 \%\left(r^{2}=0.9521\right)$ (Figure 3). The low lattice strain is due to the fact that the natural synthesis procedure (biosynthetic reaction) does not impose any constraint during the size reduction and/or formation of compound as is generally found with extensive ball milling technique, strained layer growth, etc.

\section{High-resolution transmission} electron microscopy study

Figure 4 shows the HRTEM image of n-CoF being formed using Saccharomyces cerevisiae. The micrograph clearly illustrates the nanoparticles which are almost spherical in shape (3-15 $\mathrm{nm}$ ); measurement was taken along the diameter of the particles. The lattice images of nanocrystals are cubic with $0.251 \mathrm{~nm} d$-spacing, corresponding to the (311) plane of cubic $\mathrm{CoFe}_{2} \mathrm{O}_{4}$ (Figure 4A). The particle size distribution histogram of $n-C o F$ is shown in the inset of

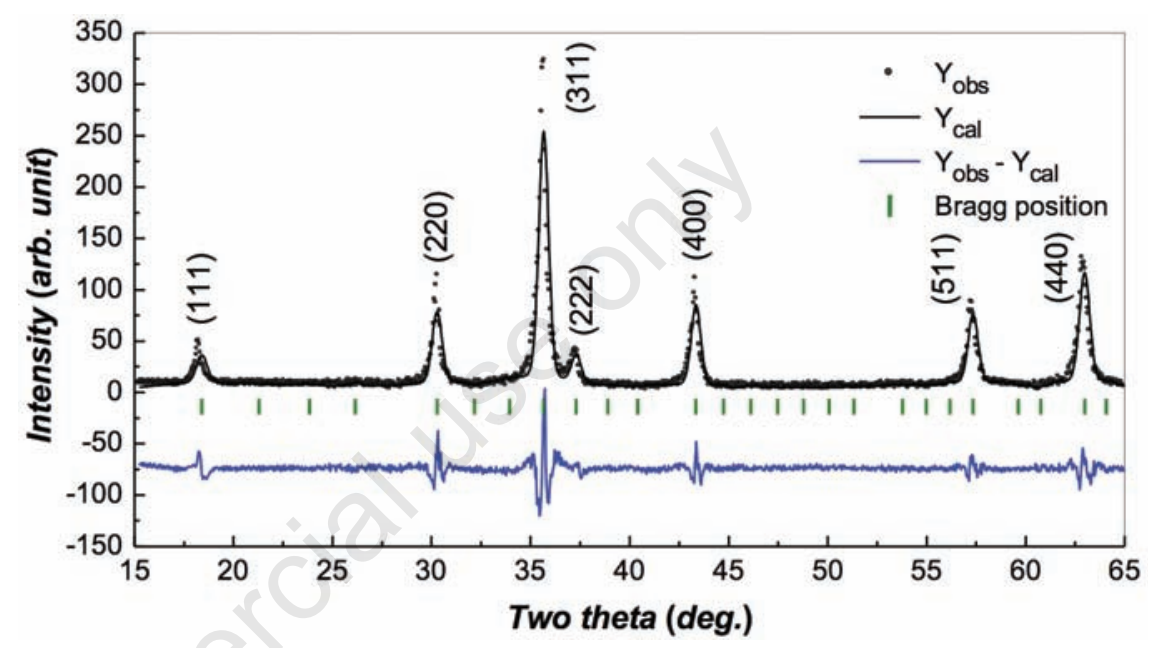

Figure 2. Rietveld refined X-ray diffraction pattern of cobalt ferrite nanoparticles.

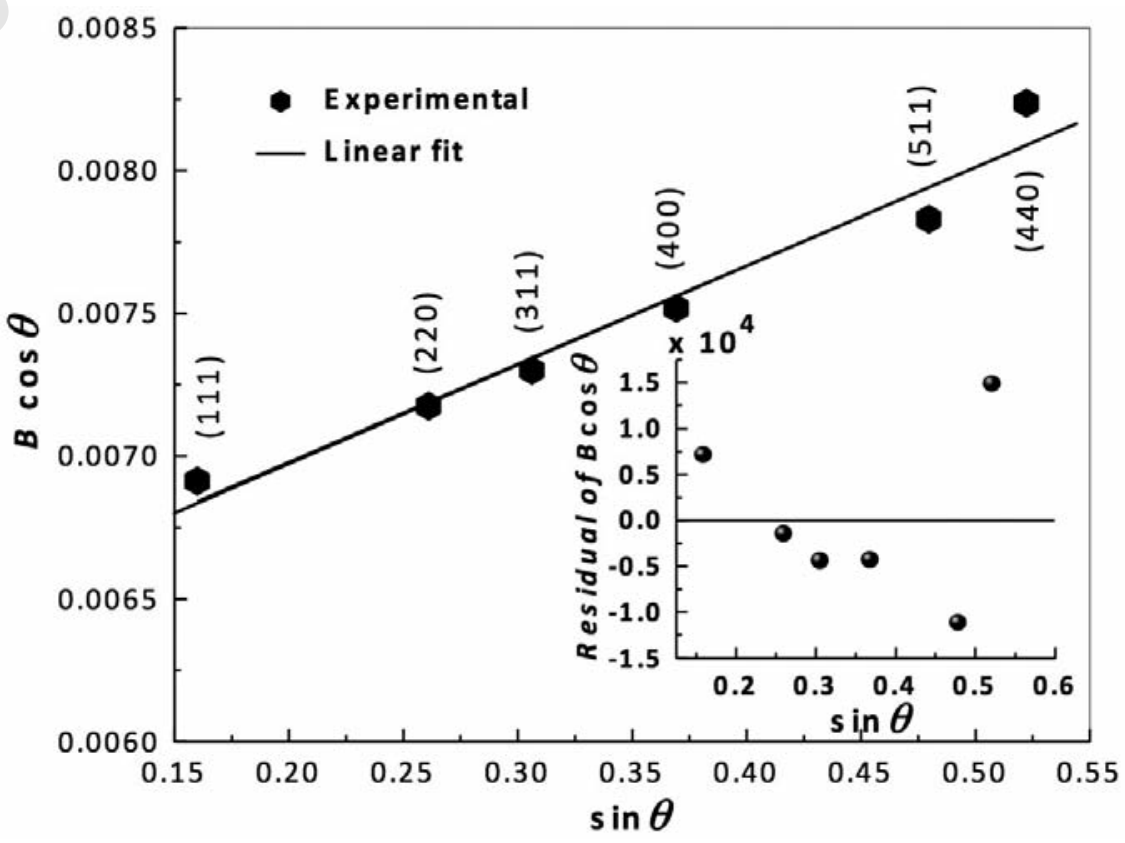

Figure 3. Williamson-Hall plot for cobalt ferrite nanoparticles. Inset: residual plot. 
Figure 4B. The difference in particle size is possibly due to the fact that the nanoparticles are being formed at different times. Average particle size was $7.23 \pm 0.06 \mathrm{~nm}$. Very little particle agglomeration could be seen in the TEM image.

\section{Fourier transform infrared spectroscopy study}

FTIR spectra of n-CoF is shown in Figure 5. It is known that the symmetry of the different lattice sites, occupied by the molecules in the crystal lattice, plays an important role in determining the nature of the vibrational modes present in the system. A small absorption peak observed at $462 \mathrm{~cm}^{-1}$ (inset Figure 5), is consistent with the ferrite skeleton for the octahedral site. ${ }^{20}$ The intense peak at $575 \mathrm{~cm}^{-1}$ in the FTIR spectrum is attributed to the M- 0 tetrahedral site in the spinel structure. This may be one of the reasons for the basic nature of the synthesized nanoparticle. ${ }^{21}$ The absence of the peaks between 1000 $1300 \mathrm{~cm}^{-1}$ and $2000-3000 \mathrm{~cm}^{-1}$ in the figure, except a peak at $1133 \mathrm{~cm}^{-1}$ corresponding to FeCo alloy ${ }^{20}$ and a peak at $1610 \mathrm{~cm}^{-1}$ belonging to stretching frequencies for the surface adsorbed - $\mathrm{OH}$ group, indicated the lack of the $\mathrm{C}-\mathrm{O}$ mode and $\mathrm{C}=\mathrm{H}$ stretching-mode of any organic sources. ${ }^{22}$ Furthermore, IR spectra are very sensitive to the presence of moisture during experimentation. A broad and strong peak can easily be seen around $3500 \mathrm{~cm}^{-1}$ which is due to free water molecules ( $\mathrm{H}_{2} \mathrm{O}$ bands) and strong stretching (antisymmetric and symmetric) modes of the $\mathrm{OH}$ group which indicates the more polar character of the surface.

\section{Magnetic study}

Figure 6 shows the M-H curve for $\mathrm{n}-\mathrm{CoF}$ at room temperature. It is observed that the $\mathrm{M}-\mathrm{H}$ curve is very slim, thus indicating the superparamagnetic behavior which is consistent with the earlier report. ${ }^{23}$ Saturation magnetization $M_{S}=41.8 \mathrm{emu} / \mathrm{g}$, coercive field $H_{C}=1300 \mathrm{e}$ and retentivity $M_{R}=13.1 \mathrm{emu} / \mathrm{g}$ were found in the sample. It is known that $\mathrm{Co}^{2+}$ has a strong preference for the octahedral site. As a result, there will be far fewer A-B magnetic ion pairs than the B-B pairs which provide only weak magnetic coupling. This might result in superparamagnetism, at least partially for the smaller particles in the sample, giving negligible coercive field and retentivivity. This appears to be the case in the present sample. The saturation magnetization is likewise small. Furthermore, the M-H curve shows that magnetization is not completely saturated till the field of $10 \mathrm{k} 0 \mathrm{e}$ used in these experiments. This could be due to the small size (quantum sizing effect) of the particles as shown in Figure 4. Besides, size distribution means there are smaller particles even when the sample contains quite a few larger particles (Figure 4B, inset). For such particles, the magnetic

moments will fluctuate due to thermal energy reducing the overall magnetization. As the applied field increases, the fluctuations are gradually reduced showing a slight increase even at high fields. ${ }^{24}$ Furthermore, it was observed that $\mathrm{n}-\mathrm{CoF}$ dispersed in an aqueous medium exhibited excellent stability and the formed colloid does not produce any precipitations even after two months, which is very important if it is applied in magnetic fluids.

\section{Probable mechanism involved in the formation of $\mathrm{CoFe}_{2} \mathrm{O}_{4}$ nanoparticles}

The chemical reactions which proceed in the culture medium may be as follows:

$$
\begin{array}{r}
\mathrm{C}_{6} \mathrm{H}_{12} \mathrm{O}_{6} \rightarrow \mathrm{C}_{2} \mathrm{H}_{5} \mathrm{OH} \rightarrow \mathrm{CH}_{3} \mathrm{CHO} \rightarrow \mathrm{CH}_{3} \mathrm{COOH} \\
\text { (Ethyl alcohol) (Acetaldehyde) (Acetic acid) }
\end{array}
$$

Table 1. The crystal data and refinement factors of cobalt ferrite nanoparticles obtained from X-ray powder diffraction data.

\begin{tabular}{lc} 
& Crystallographic data \\
Formula & $\mathrm{CoFe}_{2} \mathrm{O}_{4}$ \\
Crystal system & $\mathrm{Cubic}$ \\
\hline Space group (n.) & $P m 3 m(221)$ \\
\hline$a(\AA)$ & 8.3445 \\
$V\left(\AA^{3}\right)$ & 581.0351 \\
\hline & \\
Temperature $\left({ }^{\circ} \mathrm{C}\right)$ & 24.0 \\
Wavelength $[\mathrm{CuK} \alpha](\AA)$ & 1.5406 \\
\hline Monochromator & Graphite \\
Measuring range $\left({ }^{\circ}\right)$ & $15 \leq 2 \theta \leq 65$ \\
\hline Step $\left({ }^{\circ} 2 \theta\right)$ & 0.02 \\
Integration time $(\mathrm{s})$ & 30
\end{tabular}

\section{Program}

Function for background level

Function for peak shape

\begin{tabular}{lc}
$R_{\mathrm{p}}$ & 27.4 \\
$R_{\text {wp }}$ & 30.1 \\
$R_{\text {exp }}$ & 36.4 \\
\hline$R_{\mathrm{B}}$ & 0.0522 \\
$R_{\mathrm{F}}$ & 0.4510 \\
\hline$\chi^{2}$ & 0.6827 \\
$d$ & 1.3895 \\
\hline$Q_{D}$ & 1.8326 \\
$S$ & 0.8269 \\
\hline
\end{tabular}

$R_{\mathrm{p}}$ (profile factor) $=100\left[\Sigma\left|y_{i}-y_{i c}\right| / \Sigma\left|y_{i}\right|\right]$, where $y_{i}$ is the observed intensity and $y_{i c}$ is the calculated intensity at the $i^{\text {th }}$ step.

$R_{\text {wp }}$ (weighted profile factor) $=100\left[\Sigma \omega_{i} \mid y_{i}-y_{i c}{ }^{2} / \Sigma \omega_{i}\left(y_{i}\right)^{2}\right]^{1 / 2}$, where $\omega_{i}=1 / \sigma_{i}^{2}$ and $\sigma_{i}^{2}$ is variance of the observation.

$R_{\text {exp }}$ (expected weighted profile factor) $=100\left[(n-p) / \Sigma \omega_{i}\left(y_{i}\right)^{2}\right]^{1 / 2}$, where $n$ and $p$ are the number of profile points and refined parameters, respectively.

$R_{\mathrm{B}}$ (Bragg factor) $=100\left[\Sigma\left|I_{\text {obs }}-I_{\text {calc }}\right| / \Sigma\left|I_{\text {obs }}\right|\right]$, where $I_{\text {obs }}$ is the observed integrated intensity and $I_{\text {calc }}$ is the calculated integrated intensity.

$R_{\mathrm{F}}$ (crystallographic $R_{\mathrm{F}}$ factor) $=100\left[\Sigma\left|F_{\text {obs }}-F_{\text {calc }}\right| / \Sigma\left|F_{\text {obs }}\right|\right]$, where $F$ is the structure factor, $F=\sqrt{ }(I / L)$, where $L$ is Lorentz polarization factor.

$\chi^{2}=\Sigma \omega_{i}\left(y_{i}-y_{i c}\right)^{2}$.

$d$ (Durbin-Watson statistics) $=\Sigma\left\{\left[\omega_{i}\left(y_{i}-y_{i c}\right)-\omega_{i-1}\left(y_{i-1}{ }^{-}-y_{i c-1}\right)\right]^{2}\right\} / \Sigma\left[\omega_{i}\left(y_{i}-y_{i c}\right)\right]^{2}$.

$Q_{D}=\operatorname{expected} d$.

$S$ (goodness of fit $)=\left(R_{\text {wp }} / R_{\exp }\right)$. 


$$
\begin{aligned}
& \mathrm{Co}\left(\mathrm{NO}_{3}\right)_{2} \rightarrow \mathrm{Co}^{2+}+2 \mathrm{NO}_{3}^{1-} \\
& \mathrm{FeSO}_{4} \rightarrow \mathrm{Fe}^{2+}+\mathrm{SO}_{4}^{2-} \\
& \mathrm{Co}^{2+}+2 \mathrm{Fe}^{2+}+2 \mathrm{O}_{2}=\mathrm{CoFe}_{2} \mathrm{O}_{4}
\end{aligned}
$$

The environments of most organisms are rarely constant, so some resilience to environmental stress is essential in order for organisms to survive. ${ }^{25}$ As shown by numerous examples, knowledge obtained from yeast-based models, e.g. on the mechanism of action of and the tolerance against toxic metals, can be transferred with high efficiency to higher eukaryotes including humans. ${ }^{26,27}$ Heavy metals enter cells through channels and transporters, which normally facilitate the uptake of essential transition metal micronutrients like $\mathrm{Fe}, \mathrm{Mn}$ and $\mathrm{Zn}$, anions including phosphate and sulphate as well as sugars (glucose) and sugar derivatives. ${ }^{26,27}$ The major molecules that contribute to the detoxification mechanisms in yeast cells are glutathione (GSH) and two groups of metalbinding ligands: metallothioneins and phytochelatins, both well characterized. GSH with the structure $c$-Glu-Cys-Gly is an important tripeptide involved in various metabolic processes in bacteria, yeasts, plants and animals. The unique redox and nucleophilic properties classify this compound as a detoxificator, actively taking part in the bioreduction of and defense against free radicals and xenobiotics. GSH is also a structural unit in phytochelatin molecules, which is one of its major functions. Metallothioneins are low-molecular-weight, cysteine-rich metal-binding proteins, derived by mRNA translation. ${ }^{28}$ As and when yeast cells are challenged with a chemically unknown material (metal or its compounds), this triggers its membrane bound as well as cytosolic metabolites in order to circumvent the oxidative stress manifested in the form of reactive oxygen species (ROS) generation. The oxidoreductases harbored in the cytosol are $\mathrm{pH}$ sensitive and work in a different way. At a lower value of $\mathrm{pH}$, oxidase is activated while a higher $\mathrm{pH}$ value activates the reductase. ${ }^{29,30}$

This has been found in cases of any type of chemical stress. $\mathrm{CoFe}_{2} \mathrm{O}_{4}$ might also have triggered such a response at two distinct levels: i) quinones at the membrane level of the detoxification system of fungi which readily undergo tautomerization; ${ }^{31}$ and ii) cytoplasmic metabolite weaponry such as phytochelatin, HMT-I, glutathione, membrane bound monoxygenases, etc., which tend to detoxify such changes (Figure 7). Given this, the process could be very intricate at the molecular level where a series of stress genes are transcribed after generation of ROS. Metal or its compound, after coming in contact with microbe, is generally dealt with on the nanoscale because it is a scale natural to biological systems. Even if a material is detoxi- fied after being taken in, it is reverted back in the form of nanoscale from the cell. Once it enters the cytosol, the $\mathrm{CoFe}_{2} \mathrm{O}_{4}$ might also have triggered the family of oxygenases held in the endoplasmic reticulum, chiefly intended for cellular level detoxification through the process of oxidation/oxygenation. ${ }^{30}$ The scheme for the biosynthesis of $n-C o F$ is illustrated in Figure 7. In the light of these facts, our group had earlier reported synthesis of metallic silver, ${ }^{32}$ antimony oxide ${ }^{33}$ titanium dioxide ${ }^{34}$ and cadmium sulfide $^{35}$ Therefore, compared to the existing pro-

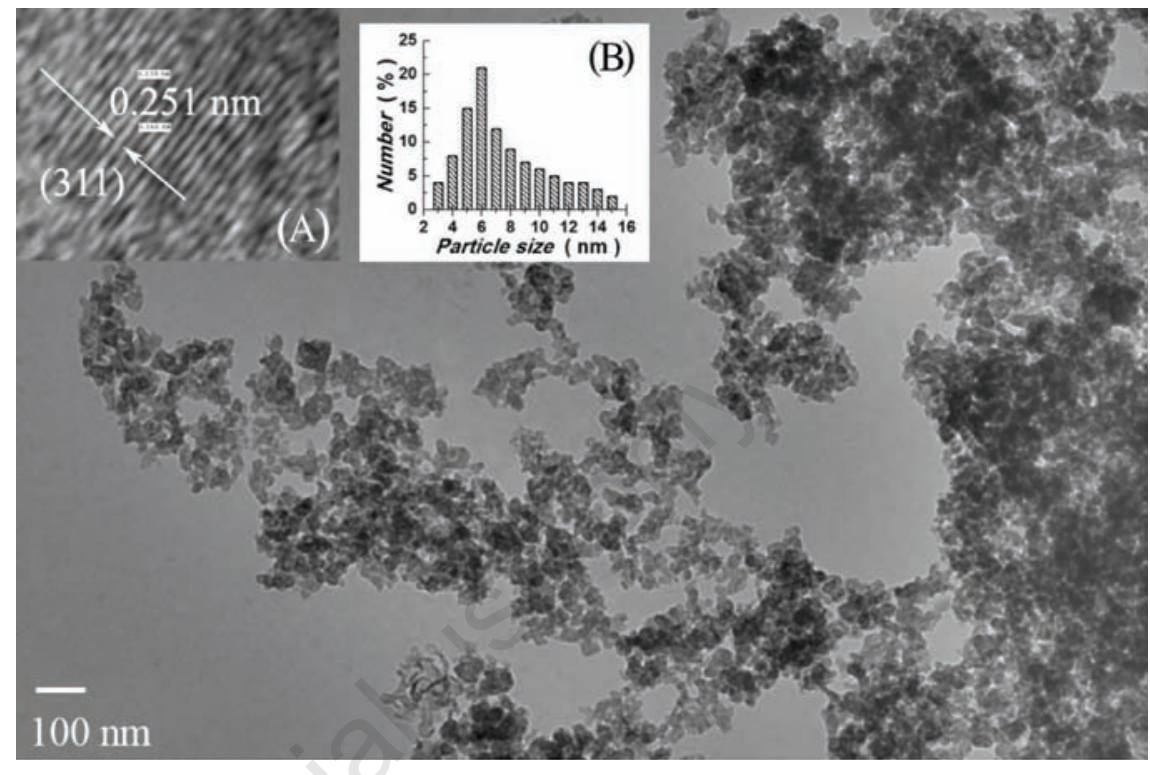

Figure 4. Transmission electron microscopy image of cobalt ferrite nanoparticles synthesized using Saccharomyces cerevisiae. (A) Lattice fringes. (B) Particle size distribution (\%).

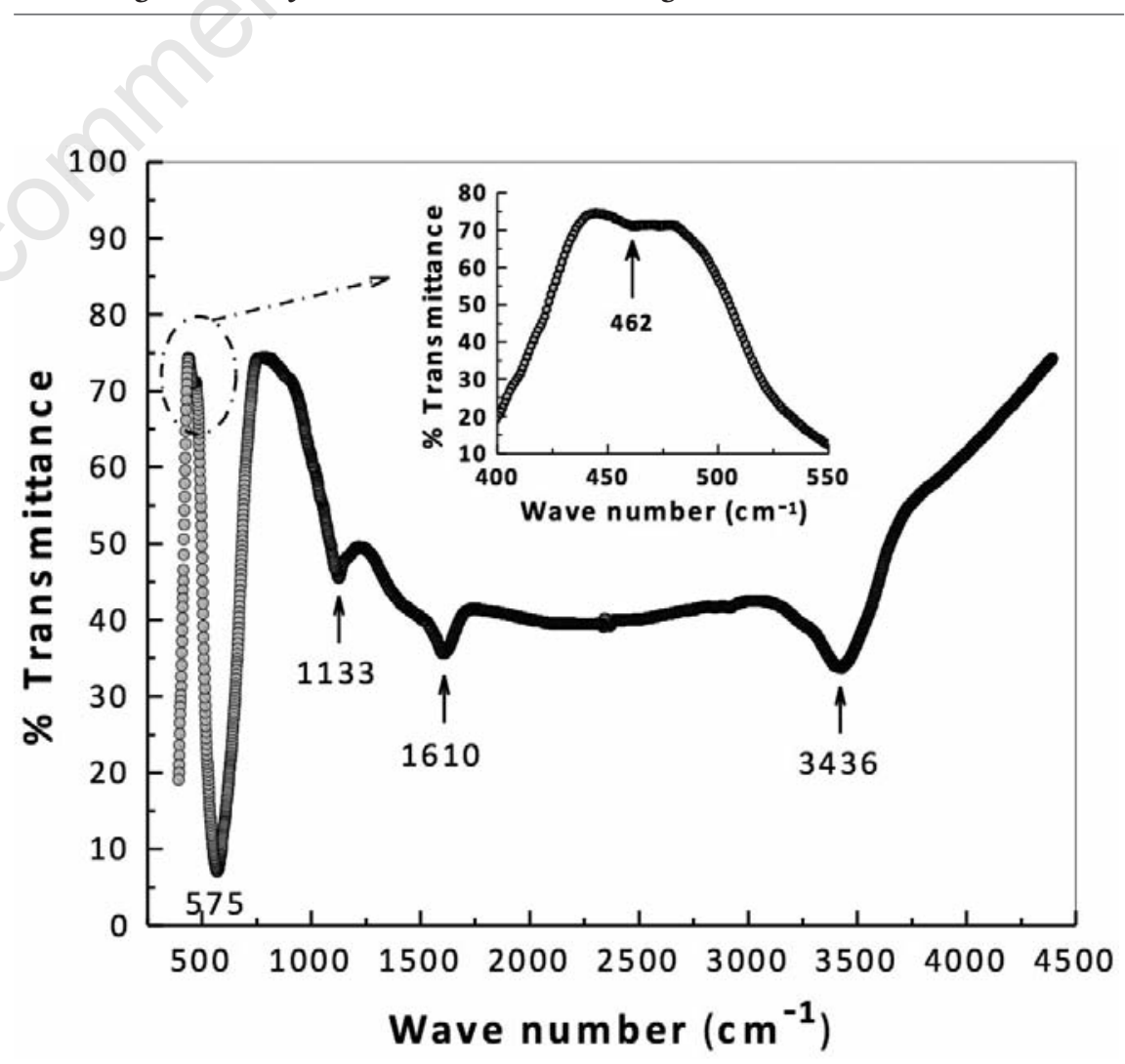

Figure 5. Fourier transform infrared spectrum of cobalt ferrite nanoparticles. 
tocols, the present procedure is less expensive, more reproducible, decisively less/non-toxic and represents a truly environmentally friendly and novel approach.

\section{Conclusions}

The present biosynthetic method, using Saccharomyces cerevisiae, is a truly environmentally friendly and cost-effective approach, capable of producing $\mathrm{CoFe}_{2} \mathrm{O}_{4}$ nanoparticles.
The average particle size estimated from TEM micrograph is $7.23 \mathrm{~nm}$. Field dependent magnetization measurement showed that the cobalt ferrite was superparamagnetic at room temperature. The maximun value of $H_{C}$ is 1300 e, which is higher than the coercitivity of its bulk counterpart. The synthesis of $\mathrm{CoFe}_{2} \mathrm{O}_{4}$ nanoparticles might have been due to a different molecular level response being generated at the membrane level as well as cytosolic level. Stress shearing systems such as metallothioneins, phytochelatin, GSH and HMT-I must have played a pivotal role.

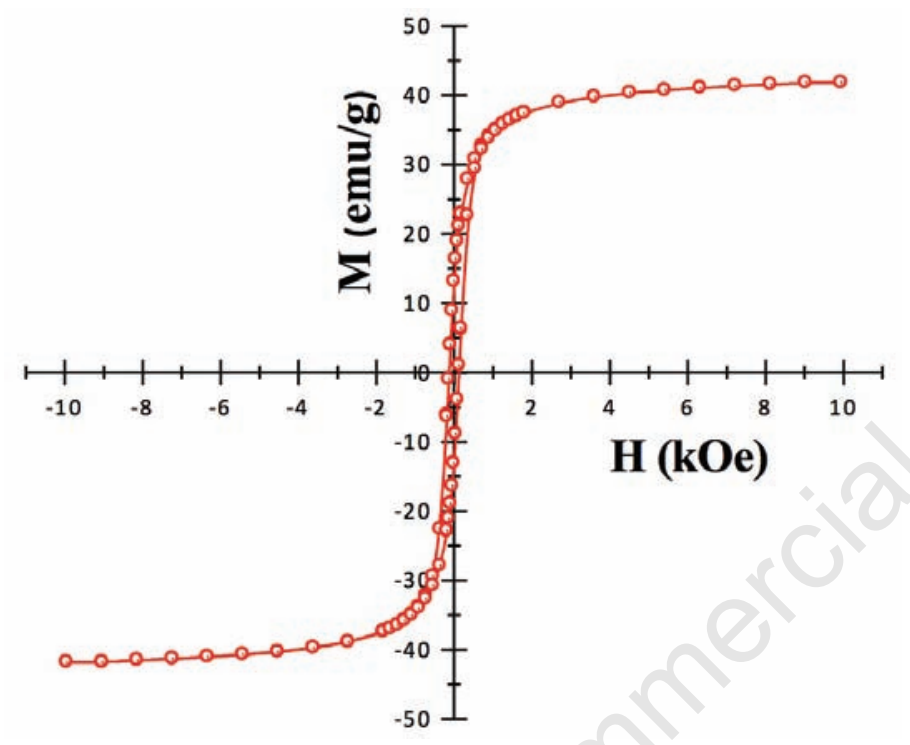

Figure 6. Magnetization curve for cobalt ferrite nanoparticles at room temperature.

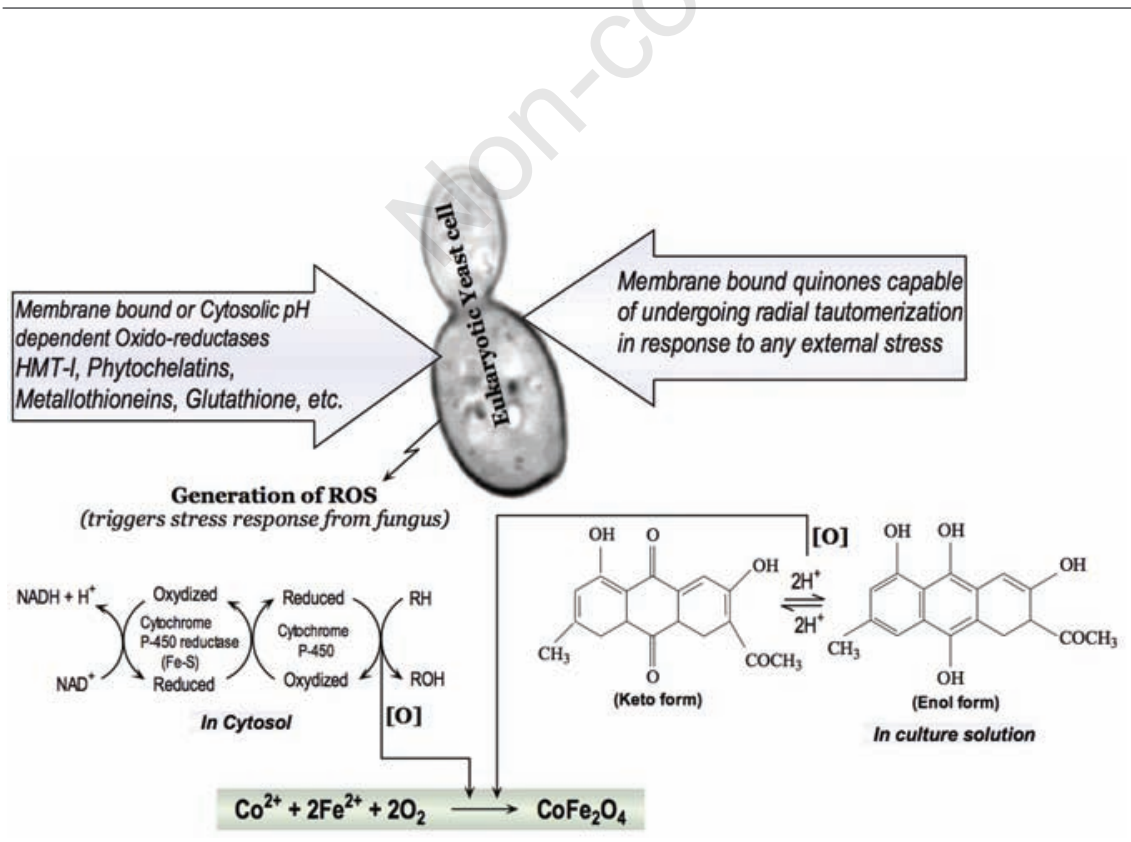

Figure 7. Schematics for the biosynthesis of cobalt ferrite nanoparticles using Saccharomyces cerevisiae.

\section{References}

1. Leslie-Pelecky DL, Rieke RD. Magnetic properties of nanostructured materials. Chem Mater 1996;8:1770-83.

2. Himpsel FJ, Ortega JE, Mankey GJ, Willis RF. Magnetic nanostructures. Adv Phys 1998;47:511-97.

3. Sugimoto M. The past, present and future of ferrites. J Am Ceram Soc 1999;82:269-80.

4. Bate G. Magnetic recording materials since 1975. J Magn Magn Mater 1991;100: 413-24.

5. Pileni MP. Magnetic fluids: fabrication, magnetic properties, and organization of nanocrystals. Adv Funct Mater 2001;11: 323-36.

6. Liu C, Zou BS, Rondinone AJ, Zhang ZJ. Reverse micelle synthesis and characterization of superparamagnetic MnFe204 spinel ferrite nanocrystallites. J Phys Chem B 2000;104:1141-5.

7. Sun C, Lee JSH, Zhang M. Magnetic nanoparticles in MR imaging and drug delivery. Adv Drug Deliv Rev 2008;60:125265 .

8. Shultz MD, Calvin S, Fatouros PP, et al. Enhanced ferrite nanoparticles as MRI contrast agents. J Magn Magn Mater 2007; 311:464-68.

9. Zhen L, He K, Xu CY, Shao WZ. Synthesis and characterization of single crystalline MnFe204 nanorods via a surfactant-free hydrothermal route. J Magn Magn Mater 2008;320:2672-5.

10. Schmid G. Nanoparticles: from theory to application. Weinheim: Wiley-VCH; 2004.

11. Jordan A, Scholz R, Wust P, et al. Endocytosis of dextran and silan-coated magnetite nanoparticles and the effect of intracellular hyperthermia on human mammary carcinoma cells in vitro. J Magn Magn Mater 1999;194:185-96.

12. Kim DH, Nikles DE, Johnson DT, Brazel CS. Heat generation of aqueously dispersed CoFe204 nanoparticles as heating agents for magnetically activated drug delivery and hyperthermia. J Magn Magn Mater 2008;320: 2390-6.

13. Mornet S, Vasseur S, Grasset F, Duguet E. Magnetic nanoparticle design for medical diagnosis and therapy. J Mater Chem 2004; 14:2161-75.

14. Wada S, Tazawa K, Furuta I, Nagae H. Antitumor effect of new local hyperthermia using dextran magnetite complex in hamster tongue carcinoma. Oral Dis 2003;9:218-23.

15. Bentivegna MF, Nyvlt M, Ferre J, et al. Magnetically textured -Fe203 nanoparticles in a silica gel matrix: optical and magneto-optical properties. J Appl Phys 1999;85:2270-8. 
16. Hocini A, Boumaza T, Bouchemat M, et al. Birefringence in magneto-optical rib waveguides made by $\mathrm{SiO2/TiO2}$ doped with -Fe203. Microelectron J 2008;39:99102.

17. Jamon D, Donatini F, Siblini A, et al. Experimental investigation on the magneto optic effects of ferrofluids via dynamic measurements. J Magn Magn Mater 2009;321:1148-54.

18. Mathew DS, Juang RS. An overview of the structure and magnetism of spinel ferrite nanoparticles and their synthesis in microemulsions. Chem Eng J 2007;129:5165.

19. McCusker LB, Von Dreele RB, Cox DE, et al. Rietveld refinement guidelines. J Appl Cryst 1999;32:36-50.

20. Rana S, Philip J, Raj B. Micelle based synthesis of cobalt ferrite nanoparticles and its characterization using Fourier transform infrared transmission spectrometry and thermogravimetry. Mater Chem Phys 2010; 124: 264-9.

21. Senapati KK, Borgohain C, Phukan P. Synthesis of highly stable CoFe204 nanoparticles and their use as magnetically separable catalyst for Knoevenagel reaction in aqueous medium. $\mathrm{J}$ Mol Catal A Chem 2011;339:24-31.
22. Kwon YJ, Kim KH, Lim CS, Shim KB. Characterization of $\mathrm{ZnO}$ nanopowders synthesized by the polymerized complex method via an organochemical route. J Ceram Proc Res 2002;3:146-9.

23. Gyergyek S, Makovec D, Kodre A, et al. Influence of synthesis method on structural and magnetic properties of cobalt ferrite nanoparticles. J Nanopart Res 2010;12: 1263-73.

24. Singh RK, Yadav A, Prasad K, Narayan A. Dependence of magnetic and structural properties of Ni0.5M0.5Fe204 ( $\mathrm{M}=\mathrm{Co}$, $\mathrm{Cu}$ ) nanoparticles synthesized by citrate precursor method on annealing temperature. Int J Engg Sci Technol 2010;2:73-9.

25. Avery SV. Molecular targets of oxidative stress. Biochem J 2011;434:201-10.

26. Tamás MJ, Labarre J, Toledano MB, Wysocki R. Mechanisms of toxic metal tolerance in yeast. In: Tamás MJ, Martinoia E, (eds). Molecular biology of metal homeostasis and detoxification: from microbes to man. Heidelberg: Springer; 2005. pp 395-454.

27. Wysocki R, Tamás MJ. How Saccharo myces cerevisiae copes with toxic metals and metalloids. FEMS Microbiol Rev 2010;34:925-51.

28. Kagi JHR. Evolution, structure and chem- ical activity of class I metallothioneins: an overview. In: Suzuki KY, Imura N, Kimura M, (eds). Metallothionein III: biological roles and medical implications. Basel: Birkhäuser Verlag; 1993. pp 29-55.

29. Nelson DN, Cox MM. Lehninger principles of biochemistry. New York: Freeman Publication; 2005.

30. Botham KM, Mayes PA. Biologic Oxidation-Harper's illustrated biochemistry. London: Lange-McGraw Hill; 2006.

31. Thomson RH. Quinone: Nature, distribution and biosynthesis. In: Goodwin TW, (ed). Chemistry and biochemistry of plant pigments. New York: Academic Press; 1976. pp 527-59.

32. Prasad K, Jha AK, Kulkarni AR. Yeast mediated synthesis of silver nanoparticles. Int J Nanosci Nanotechnol 2008;4:17-21.

33. Jha AK, Prasad K, Prasad K. A green lowcost biosynthesis of Sb203 nanoparticles. Biochem Eng J 2009;43:303-06.

34. Jha AK, Prasad K, Kulkarni AR. Synthesis of TiO2 nanoparticles using microorganisms. Colloid Surf B Biointerfaces 2009; 71:226-9.

35. Prasad K, Jha AK. Biosynthesis of CdS nanoparticles: an improved green and rapid procedure. J Colloid Interface Sci 2010;342:68-72. 